\title{
NEOCLASSICAL TEARING MODES
}

\author{
H.R.Wilson \\ York Plasma Institute, Department of Physics, University of York, Heslington, York, YO10 5DQ U.K. \\ howard.wilson@york.ac.uk
}

\begin{abstract}
Tearing modes often limit the performance of tokamak plasmas, because the magnetic islands which they generate lead to a loss of confinement, or even a disruption. A particularly dangerous instability is the neoclassical tearing mode, which can grow to a large amplitude because of the amplification effect that the bootstrap current has on an initial 'seed' magnetic island. This paper will address the mechanisms which dominate the neoclassical tearing mode evolution, and thereby identify possible control techniques.
\end{abstract}

\section{INTRODUCTION}

The good confinement of the tokamak is achieved because to leading order the ions and electrons follow the magnetic field lines, which in turn lie on toroidally symmetric, nested magnetic flux surfaces. However, there are a number of plasma instabilities which can modify the magnetic geometry and so lead to a reduction in confinement and a loss of plasma stored energy. In this paper we shall concentrate on a particular type of instability, the tearing mode, and explore its consequences for tokamak performance. One consequence of the tearing mode instability is that the plasma adopts a new, non-symmetric equilibrium (or, if the instability is particularly violent, the plasma can be lost altogether in a disruption). This new equilibrium is characterised by a chain of magnetic islands, and field lines can migrate radially around these over a distance comparable to the island width. The result is that the radial particle and energy flux is enhanced in the regions where the magnetic islands form, and the overall confinement is degraded (eg the central plasma temperature is reduced). For this reason, understanding the causes of tearing modes is an important part of tokamak physics research, and this paper provides a brief review of the progress made in our understanding, and the gaps that remain.

We shall begin in Section II with a brief summary of the basic properties of tearing modes, and provide a simple derivation of the 'classical' (Rutherford) tearing mode evolution equation ${ }^{1}$. Then in Section III we shall address a number of other mechanisms which can contribute to the tearing mode evolution in toroidal plasmas to derive, heu- ristically, the so-called modified Rutherford equation for neoclassical tearing modes (NTMs). In Section IV we shall explore the experimental evidence for neoclassical tearing modes and make comparisons with the theory. Finally, in Section V, we shall consider some of the control methods which have been proposed, largely motivated by our theoretical understanding of these instabilities.

\section{CLASSICAL TEARING MODE PHYSICS}

Let us begin by introducing some of the terminology associated with tearing mode physics, broadly following Ref [2]. To simplify the geometry, let us take an annulus of toroidal plasma with major radius $R$ (say between minor radii $r=r_{1}$ and $r=r_{2}$ ), and cut this open in the toroidal and poloidal directions to form the plasma slab shown in Fig 1. We have placed an island chain at the radial position $r=r_{s}$ and indicated the positions of the so-called X-points and O-points of the island. It is conventional to define the mode structure in terms of the dominant Fourier components of the island; the case shown in Fig 1 has poloidal mode number $m=2$ and toroidal mode number $n=1$. Note that the dashed line connecting the island O-points is approximately a line of symmetry in the large aspect ratio approximation of the tokamak. Thus the island magnetic geometry can be defined in terms of three coordinates: the radial variable, $r$, the poloidal angle, $\theta$, and a new helical angle, $\xi$, which is directed along a line perpendicular to

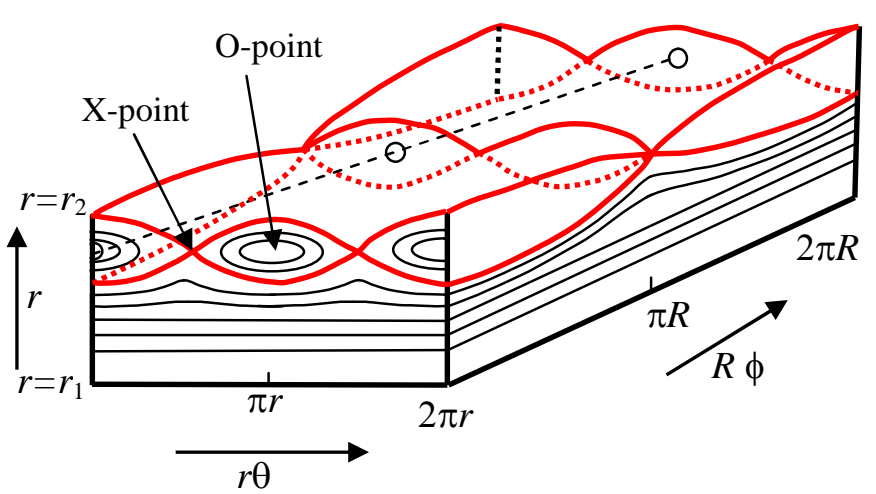

Fig. 1. A toroidal annulus of plasma showing flux surfaces forming magnetic islands. The annulus has been cut along the poloidal $(\theta)$ and toroidal $(\phi)$ directions and opened out. 
that connecting the island O-points

$$
\xi=\theta-\frac{n}{m} \phi
$$

Again adopting a large aspect ratio approximation, we see that the component of magnetic field in the helical direction is given by

$$
B_{h}=B_{\theta}\left(1-\frac{n}{m} q(r)\right)
$$

where $B_{\theta}$ is the poloidal component of the magnetic field and $q(r)$ is the safety factor. The role of the tearing mode instability is to provide the radial component of magnetic field required to generate a magnetic island. Denoting this by $\delta B=B_{r} \sin m \xi$, and noting that a field line will follow a trajectory given by

$$
\frac{d r}{r_{s} d \xi}=\frac{\delta B}{B_{h}}
$$

we see that the radial excursion of field lines is negligible unless $B_{h}$ is small (we consider that the radial field generated by the tearing mode is typically much smaller than the equilibrium magnetic fields imposed in the tokamak by the machine operator). Thus the largest radial excursions are experienced at the radial position where $q=m / n$; that is, island chains form on rational surfaces. Taylor expanding $q$ about the rational surface $r=r_{s}$, then we can use Eq (2) in $\mathrm{Eq}(3)$ to derive the following equation for the field lines:

$$
\Omega=\frac{2 x^{2}}{w^{2}}-\cos m \xi
$$

where $x=r-r_{s}, \Omega$ is a flux surface label (a constant of the integration) and

$$
w=2\left(\frac{r q B_{r}}{m B_{\theta} d q / d r}\right)^{1 / 2}
$$

is the island half-width. Note that $\Omega=1$ defines the island separatrix, $-1<\Omega<1$ defines flux surfaces inside the island and $\Omega>1$ defines flux surfaces outside the island.

Having described the geometry and introduced the essential terminology, we are now in a position to describe some of the basic theoretical principles behind tearing modes. The theory can be broadly categorised into linear and non-linear theory. We shall be concerned with larger magnetic islands, so that the non-linear theory is the appropriate one to adopt here. Above, we characterised the perturbation in terms of the radial magnetic field it produced; in fact we shall find it more convenient to instead use the flux function, $\psi$. Thus we define the perturbed flux

$$
\psi=\tilde{\psi} \cos m \xi \quad B_{r}=\frac{m \tilde{\psi}}{r R}
$$

where $\tilde{\psi}$ is related to $B_{r}$ and is assumed to vary only slowly with radius over the island width length scale. In terms of $\psi$, the total magnetic field is given by

$$
\mathbf{B}=f(r) \nabla \phi+\nabla \phi \times \nabla(\Psi+\psi),
$$

where $f(r)=R B_{\phi}$ and $\Psi(r)$ is the poloidal magnetic flux. Let us restrict consideration to small magnetic islands whose width is much less than the tokamak minor radius. Then the current perturbation is small, and we are justified in assuming that $\psi$ varies only slowly with $r$. For islands whose width is much less than their length, Ampere's law relates $\psi$ to the current density perturbation parallel to the magnetic field, $J_{\|}$:

$$
\frac{1}{R} \frac{d^{2} \psi}{d r^{2}}=\mu_{0} J_{\|},
$$

Another condition on the validity of this expression is that the perturbed current is localised about the island region so that, although $d \psi / d r$ is small, it changes rapidly in a narrow region in the vicinity of the island so that its second derivative need not be small. Making use of this we integrate across the island region from $r=-l$ to $r=l$, where $r_{s}>>l>>w$ is assumed. In addition we define a parameter which characterises the jump in $d \psi / d r$ across the island, conventionally denoted by the symbol $\Delta^{\prime}$ :

$$
\Delta^{\prime}=\lim _{l \rightarrow \infty} \frac{1}{\psi}\left[\left.\frac{d \psi}{d r}\right|_{r=l}-\left.\frac{d \psi}{d r}\right|_{r=-l}\right]
$$

As a result we arrive at the following basic equation for tearing mode evolution:

$$
\Delta^{\prime} \tilde{\psi}=2 \mu_{0} R \int_{-\infty}^{\infty} d x \oint d \xi J_{\|} \cos m \xi
$$

The next task is to determine the perturbed current. Note that it is only the current parallel to the magnetic field that contributes to the island evolution equation. The simplest model is that considered by Rutherford ${ }^{1}$, in which the only contribution to $J_{\|}$comes from the induced current associated with island growth. Thus, for an island which is evolving, so that $\psi$ has a time-dependence, an electric field proportional to $d \psi / d t$ is generated parallel to the magnetic field (note that $\psi$ is proportional to the component of the 
perturbed vector potential in the magnetic field direction). This gives rise to a current via Ohm's law:

$$
\eta J_{\|}=\frac{\partial \tilde{\psi}}{\partial t} \cos m \xi-\nabla_{\|} \varphi
$$

where $\eta$ is the plasma resistivity, $\varphi$ is the electrostatic potential and $\nabla_{\|}$is the derivative along the perturbed magnetic field lines of the island.

It is worthwhile spending a little time considering the $\nabla_{\|}$operator, which is defined as

$$
\nabla_{\|} \equiv \frac{\mathbf{B} \cdot \nabla}{B}=\left.\frac{1}{R q} \frac{\partial}{\partial \theta}\right|_{\Omega, \xi}+\left.k_{\|} \frac{\partial}{\partial \xi}\right|_{\theta, \Omega}
$$

where $k_{\|}=-m x / r L_{s}$ and $L_{s}=R q / s$, with $s=(r / q)(d q / d r)$ being the magnetic shear. A useful procedure is to define an average over the two angles, $\theta$ and $\xi$, which annihilates the $\nabla_{\|}$operator. We shall indicate this averaging procedure by angled brackets, defined as

$$
\langle\cdots\rangle \equiv\left\{\begin{array}{cc}
\frac{\oint d \theta \oint d \xi \frac{\cdots}{\sqrt{\Omega+\cos \xi}}}{\oint d \theta \oint d \xi \frac{1}{\sqrt{\Omega+\cos \xi}}} & \Omega>1 \\
\sum_{\sigma} \sigma \frac{\xi_{b}}{\oint \int_{-\xi_{b}}^{\xi_{b}} d \xi \frac{\cdots}{\sqrt{\Omega+\cos \xi}}} & -1<\Omega<1 \\
\oint \int_{-\xi_{b}} d \xi \frac{1}{\sqrt{\Omega+\cos \xi}} &
\end{array}\right.
$$

where $\cos \xi_{b}=-\Omega$ and $\sigma=x /|x|$. We can now use this averaging operator to eliminate $\varphi$ from Eq (11) as follows. First we note that we expect $J_{\|}$to be a function only of $\Omega$, due to the fact that we neglect particle drifts perpendicular to the magnetic field for the present (so that perpendicular currents must also be absent), and therefore we must satisfy $\nabla \cdot \mathbf{J}=\nabla_{\|} J_{\|=0}$. Thus, we arrive at the result

$$
J_{\|}=\frac{1}{\eta} \frac{\partial \tilde{\psi}}{\partial t}\langle\cos m \xi\rangle
$$

and substitution of this into Eq (10), together with Eq (5), yields the classical Rutherford tearing mode evolution equation:

$$
a_{1} \tau_{r} \frac{d w}{d t}=r_{s}^{2} \Delta^{\prime}
$$

where $\tau_{r}=\mu_{0} r_{s}{ }^{2} / \eta$ is the current diffusion time and $a_{1}=0.82$ is associated with the island geometry. Note that this equation predicts that an island will grow linearly in time provided $\Delta^{\prime}>0$, at least initially when the island is sufficiently small that $\Delta^{\prime}$ is independent of $w$. Clearly the parameter $\Delta^{\prime}$ is important for the stability of tearing modes, and it is therefore useful to say a few more words about its physical significance, and how it is determined.

Recall that we have assumed that $\psi$ is approximately independent of $r$ in the vicinity of the island, and so far we have only solved for $\psi$, or equivalently $w$, in that region. Away from the island region, two simplifying approximations can be made: (1) the plasma response is linear, and (2) resistivity is unimportant. Thus, away from the island region the equations of linear ideal magnetohydrodynamics (MHD) can be used to evaluate $\psi(r)$ (note that over the longer length scales across the plasma minor radius, the $r$ dependence of $\psi$ cannot be neglected, and indeed is calculated from the ideal MHD equations). Applying appropriate boundary conditions at the plasma edge and centre, and integrating the MHD equations from the centre out to the rational surface, and from the edge into the rational surface, one can calculate $\psi(r)$ over the full plasma region, taking $\psi$ to be continuous at the island rational surface. In general, one will find that this solution will have a discontinuous gradient at the rational surface, and from this one can calculate $\Delta^{\prime}$ from the ideal region using Eq (9), but replacing $-l$ with the limit as $r \rightarrow r_{s}$ from below, and $+l$ with the limit as $r \rightarrow r_{s}$ from above. This is basically a matching condition between the solution for $\psi$ in the ideal MHD region and that in the island region. Thus we see that $\Delta^{\prime}$ is a property of the global plasma equilibrium, and in the limit of small islands $\left(w<<r_{s}\right)$ is not influenced by the presence of the island itself. Indeed, it can be shown that $\Delta^{\prime}$ represents the free energy available in the plasma current density distribution to drive the tearing mode. In the following sections we will see how other effects can modify the evolution of tearing modes, but these are different from the $\Delta^{\prime}$ drive in that they originate from the island region itself.

\section{THE MODIFIED RUTHERFORD EQUATION}

In the previous section we considered only the inductive contribution (due to island growth) to the perturbed current, $J_{\|}$. In this section we consider a number of other contributions, which together constitute the ingredients of the so-called neoclassical tearing mode (NTM).

Let us begin by considering the most important element: the perturbed bootstrap current ${ }^{3,4}$. The bootstrap current is a current which flows along the tokamak magnetic field lines due to the combined effect of the trapped particles and the density and temperature gradients which exist. We do not go into the details of this current here, but it suffices to know that the bootstrap current is proportional to a linear combination of density and temperature gra- 
dients, and requires the plasma to be in a low (so-called 'banana' or 'plateau') collisionality regime so that trapped particles can perform a complete orbit before being detrapped by collisions. For our purposes it is sufficient to use a simple model for the bootstrap current, which we express in the form:

$$
J_{b s}=-2.44 \frac{\sqrt{\varepsilon}}{B_{\theta}} \frac{d p}{d r}
$$

This expression is accurate in the limit of small inverse aspect ratio, $\varepsilon$, and zero temperature gradient ( $p$ denotes the plasma pressure).

The main reason for a perturbation in the bootstrap current in the vicinity of the island is due to the island's effect on the plasma pressure there. Suppose that at some initial time there exists a magnetic island. There is rapid parallel transport along field lines so that the pressure is approximately a flux surface quantity; this means that in the absence of heat and particle sources inside the island, the pressure gradient tends to be removed from inside the island. From Eq (16) we therefore see that the bootstrap current is removed from inside the island, whilst outside (where a pressure gradient is still maintained across the flux surfaces) the bootstrap current remains. Thus there is a 'hole' in the bootstrap current which exists around the island O-points; ie there is an additional contribution to $J_{\|}$ which has the required $\cos m \xi$ component to contribute to the island evolution in Eq (10). Thus, if we now combine this contribution with the inductive contribution, Eq (14), and substitute the total $J_{\|}$into Eq (10) (using Eq (5) for the island width in place of $\psi$ ), we find:

$$
a_{1} \frac{\tau_{r}}{r_{s}^{2}} \frac{d w}{d t}=\Delta^{\prime}+a_{2} \sqrt{\varepsilon} \frac{\beta_{\theta}}{w} \frac{L_{q}}{L_{p}}
$$

We have introduced a new numerical factor $a_{2}$, which originates from the integral over space, the poloidal beta, $\beta_{\theta}=2 \mu_{0} p / B_{\theta}{ }^{2}, L_{q}^{-1}=d \ln q / d r$ and $L_{p}{ }^{-1}=-d \ln p / d r$. Note that in normal tokamak situations $L_{q} / L_{p}>0$ and therefore the bootstrap current term usually contributes a drive for the tearing mode (a notable exception is the case of reverse shear discharges, where $L_{q}<0$ ). Indeed, for sufficiently small island widths the bootstrap term is the dominant one, so that even in situations when the plasma is stable to the classical tearing mode, the effect of the bootstrap current is to drive it unstable. In such cases the instability is called a neoclassical tearing mode.

Let us suppose that we are in this neoclassical tearing mode instability regime, so that $\Delta^{\prime}<0$. It is useful to plot $d w / d t$ as a function of $w$, and this is shown in Fig 2. There

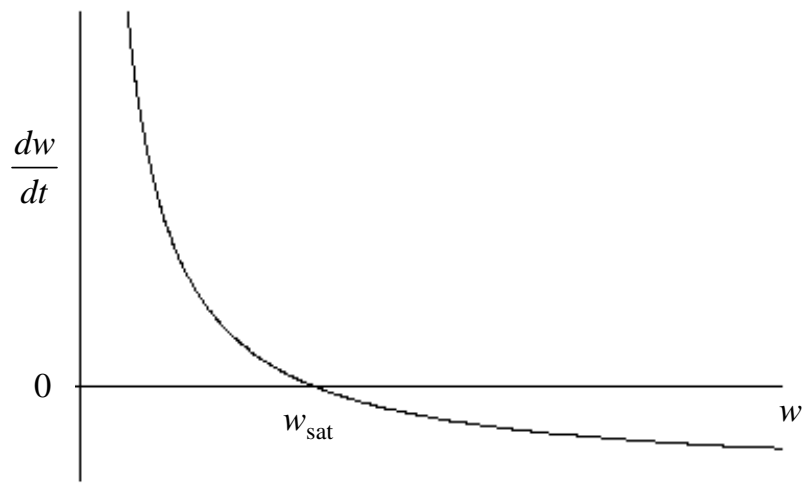

Fig. 2. The island growth as a function of the width, from Eq (17) indicating the saturated island width solution at $w=w_{\text {sat }}$.

is an important value of $w=w_{\text {sat }}$ for which $d w / d t=0$ : for $w<w_{\text {sat }}, d w / d t>0$, so the island will grow until $w=w_{\text {sat }}$; for $w>w_{\text {sat }}, d w / d t<0$, so the island will shrink until $w=w_{\text {sat }}$. Thus we see that $w=w_{\text {sat }}$ is a stable point, corresponding to the saturated island width that the neoclassical tearing mode will evolve towards. We can use Eq (17) to derive:

$$
\frac{w_{\mathrm{sat}}}{r_{s}}=a_{2} \sqrt{\varepsilon} \frac{\beta_{\theta}}{\left(-r_{s} \Delta^{\prime}\right)} \frac{L_{q}}{L_{p}}
$$

In order to gain an order of magnitude estimate of the effect, let us further suppose that $L_{q} \sim L_{p}$ and that $r_{s} \Delta^{\prime} \sim-2 m$ (which is correct in the asymptotic limit of large $m$ ); then we find that

$$
\frac{w_{\mathrm{sat}}}{r_{s}} \sim \frac{\beta_{\theta}}{2 m}
$$

Equation (19) illustrates why these modes are so dangerous: as we increase $\beta_{\theta}$ the island will grow, leading to an ever increasing degradation in confinement; eventually a situation would be reached where all the heating power which is put into the plasma is immediately flushed out by the island, and it will be impossible to increase $\beta_{\theta}$ further. In this sense, the NTM provides a 'soft' $\beta$-limit. However, particularly for low $m$ modes, we see that Eq (19) predicts that island sizes can become comparable to the minor radius of the tokamak: then we would expect the plasma to respond violently, and terminate in a disruption.

If Eq (17) represented the full story, then the future of the tokamak would be exceedingly bleak, and indeed it would not have enjoyed the success it has had, particularly in recent years. The point is that, according to Eq (17), all neoclassical tearing modes which have a rational surface in the plasma would be unstable and the confinement would be completely wrecked. This clearly is not the case, 
and so there must be more to the story. Indeed there are additional effects which are important when the island size is very small. For such small islands the theory is seriously complicated by both finite particle orbit width effects and finite radial transport effects. To illustrate this, note that the theory used to calculate the bootstrap current expression given in $\mathrm{Eq}$ (16) is based on an expansion in the ratio of ion banana width to the equilibrium length scales, assumed small. Clearly, then, for islands whose width is of order the ion banana width, Eq (17) may be flawed. Indeed, all rigorous analytic calculations of the modified Rutherford equation to date rely on an expansion in the ratio of the ion banana width to the island width: this therefore sets the scale at which the theory must be questioned. Let us now look briefly at two additional effects which may be important for such small islands.

We begin with the effects of radial diffusion ${ }^{5,6}$. Recall that we made the statement that the pressure gradients would be removed from inside the island region. This is a statement that the parallel transport effects dominate the radial diffusion. For arguments sake, let us consider a model for the electron heat transport (the particle transport is further complicated by the requirement that we expect quasi-neutrality to be maintained and the parallel transport would be dominated by sound waves). In steady state, and in the absence of any heat sources, we expect $\nabla \cdot \mathbf{Q}=0$, where $\mathbf{Q}$ is the heat flux. Suppose the heat flux parallel to the field lines is given by $\mathrm{Q}_{\|}=-n \chi_{\|} \nabla_{\|} T$ and that perpendicular to the field lines is $\mathrm{Q}_{\perp}=-n \chi_{\perp} \nabla_{\perp} T$ where $\chi_{\|}$and $\chi_{\perp}$ are the thermal diffusivities parallel and perpendicular to the magnetic field, respectively, $n$ is the density and $T$ is the temperature. Taking these diffusivities to be approximately constant over the island width length scale of interest, we deduce

$$
\nabla \cdot \mathbf{Q}=n \chi_{\|} \nabla_{\|}^{2} T+n \chi_{\perp} \nabla_{\perp}^{2} T=0
$$

Now if the perpendicular transport can be neglected, then Eq (20) clearly provides the result that the temperature is constant on a field line (and it then follows that it must be constant inside the island). Suppose we now consider the conditions under which the perpendicular transport effects cannot be neglected. It is easiest to assume that $T$ is independent of $\theta$, ie $T=T(\Omega, \xi)$, and then the parallel operator can be taken to be of order $m w /\left(R q L_{q}\right)$ (see Eq (12) and note that the relevant length scale in $k_{\|}$is $x \sim w$ ). For the perpendicular gradients, the relevant length scale is again $w$, and so we deduce that the radial transport term will compete with the parallel transport term when

$$
\frac{m^{2} w^{2}}{R^{2} q^{2} L_{q}^{2}} \chi_{\|} \sim \frac{\chi_{\perp}}{w^{2}}
$$

that is, for a sufficiently small magnetic island. Rearranging Eq (21) we can therefore deduce a critical island width, $w_{\chi}$, below which the pressure is not flattened across the island, and therefore the drive for the NTM is reduced:

$$
w_{\chi}=\sqrt{\frac{R q L_{q}}{m}}\left(\frac{\chi_{\perp}}{\chi_{\|}}\right)^{1 / 4}
$$

[Note that in hot, collisionless plasmas, free streaming dominates the parallel transport, resulting in a balance $k_{\|} \mathrm{V}_{\|} \sim \chi_{\perp} \nabla_{\perp}{ }^{2}$, and a different scaling for $w_{\chi}$ ]. To estimate the size of $w_{\chi}$ and how it scales with plasma parameters is difficult because this needs knowledge of the perpendicular heat diffusivity in the plasma, and this is not wellunderstood. If one puts in neoclassical heat diffusivity, then one obtains a very small value of the order $1 \mathrm{~mm}$ : clearly the NTM model we have described is not appropriate at such small scale lengths, when finite Larmor radius effects will inevitably play a role. However, we know that in tokamaks the perpendicular heat flux is larger than the neoclassical prediction because of the plasma turbulence. As one possible model for this, let us assume that the transport has a gyro-Bohm scaling, ie $\chi_{\perp} \sim \rho_{i}{ }^{2} \mathrm{v}_{\mathrm{thi}} / r$, where $\rho_{j}$ is the Larmor radius and $v_{\text {thj }}$ is the thermal velocity ( $j$ labels ions or electrons). Taking a collisional model for the parallel diffusivity, $\chi_{\|} \sim v_{\text {the }}{ }^{2} / v_{\mathrm{e}}$, where $v_{\mathrm{e}}$ is the electron collision frequency, we then have the estimate:

$$
w_{\chi} \sim \sqrt{\frac{L_{q} \rho_{i}}{m}} v_{* e}^{1 / 4} q^{1 / 4}\left(\frac{\varepsilon m_{e}}{m_{i}}\right)^{1 / 8}
$$

where $m_{j}$ and $v_{* j}$ are the mass and collisionality of species $j$, respectively. If we take typical tokamak parameters, then we find that this predicts a value in the region $w_{\chi} \sim 1 \mathrm{~cm}$. This value puts us above the length scales where Larmor radius effects are important, but is typical of the ion banana width in a tokamak, and therefore we remain in a regime where finite orbit width effects need to be taken into account. [Note that the parallel transport of density and ion heat is slower that that of the electron heat, and thus $w_{\chi}$ would be somewhat larger for these quantities.]

Let us now consider finite orbit width effects. There is no simple model to describe these, and therefore we will not attempt to reproduce the analysis here, but instead restrict ourselves to a discussion of the origin of the effect. Interested readers can consult the reference list for the more detailed theory, which is an evolving subject ${ }^{7-14}$. For small magnetic islands with width comparable to the ion banana width, the ions and electrons respond differently to the perturbed magnetic surfaces. For the electrons, the 
parallel streaming (ie the $\mathrm{v}_{\|} \nabla_{\|}$term in the kinetic equation) dominates their response, and the electron distribution function will adjust so that, to leading order, it will be constant along the perturbed field lines. In contrast, for the ions the $\boldsymbol{E} \times \boldsymbol{B}$ drift dominates their response. Clearly the ion density must be a flux surface quantity if the electron density is (to satisfy quasi-neutrality) and therefore the $\boldsymbol{E} \times \boldsymbol{B}$ drift must be strongest along the perturbed flux surfaces. This, in turn, means that an electrostatic potential must be generated which is constant on the island flux surfaces. Away from the island (ie a few island widths away) both the electron and ion distribution functions are unaffected by the island, and therefore this electrostatic potential is localised around the island.

Having established that an electrostatic potential is an essential feature of any small scale island, let us now consider the more detailed consequences of this. The trapped ions will execute their banana orbits, and in doing so will experience an average of the potential over these orbits. The electrons, on the other hand, have a much narrower banana orbit, and they will experience the local potential. The consequence of this is that the $\boldsymbol{E} \times \boldsymbol{B}$ drifts of the two species will differ, and therefore a current perpendicular to the magnetic field will be generated. This is the neoclassical polarisation current. We noted below Eq (10) that only a current parallel to the magnetic field can affect the island evolution. However, one finds that the divergence of this perpendicular current is not zero and therefore a small electric field is generated, directed along magnetic field lines. This accelerates the electrons to generate a parallel current (the sum of this parallel current and the perpendicular current is divergence-free), and this does contribute to the island evolution. An additional feature of the neoclassical polarisation current is that when the ion collision frequency is sufficiently high, ie $v_{\mathrm{i}} / \varepsilon \omega>1$ ( $\omega$ is the island propagation frequency in the frame where the electric field far from the island is zero), the drift information carried by the trapped ions is communicated to the passing ions, leading to a large amplification of the polarisation current ${ }^{9,10}$.

If one works through the algebra, one finds that this polarisation current contributes an additional term to the modified Rutherford equation, known as the polarisation term. A final point to note is that this polarisation term depends on the island propagation frequency $\omega$, and can be either stabilising or destabilising. This complicates matters because additional, uncertain, physics related to plasma dissipation processes (eg viscosity or Landau damping) needs to be introduced in order to determine $\omega$, and here the theory is as yet incomplete ${ }^{15}$. What is generally assumed, and this will suffice for our purpose, is that the mode frequency is such that the polarisation current provides a stabilising effect (without this assumption it is dif- ficult to interpret the experimental data, which we come to in the next section). The result is our final expression for the modified Rutherford equation, which becomes:

$$
\begin{gathered}
a_{1} \frac{\tau_{r}}{r_{s}^{2}} \frac{d w}{d t}=\Delta^{\prime}+a_{2} \sqrt{\varepsilon} \frac{\beta_{\theta}}{w} \frac{L_{q}}{L_{p}} \frac{w^{2}}{w^{2}+w_{\chi}^{2}} \\
-a_{3} g\left(\varepsilon, v_{i}\right)\left(\frac{\rho_{b i}}{w}\right)^{2}\left(\frac{L_{q}}{L_{p}}\right)^{2} \frac{\beta_{\theta}}{w} \\
g\left(\varepsilon, v_{i}\right)=\left\{\begin{array}{cc}
1.64 \varepsilon^{1 / 2} & v_{i} / \varepsilon \omega<<1 \\
\varepsilon^{-1} & v_{i} / \varepsilon \omega>>1
\end{array}\right.
\end{gathered}
$$

$\rho_{b i}$ is the ion banana width and $a_{3}$ is a third numerical coefficient associated with the spatial integral (which appears in Eq (10)) and the value of $\omega$. Equation (24) can be derived using drift-kinetic theory, provided the island width is larger than the ion banana width and $w_{\chi} \rightarrow 0^{9}$. We shall assume that the expression actually holds for island widths down to the ion banana width, but stress that as yet there is no theoretical justification for this assumption. Such a justification is a challenging task, which can probably only be addressed through large scale computational modelling ${ }^{12}$.
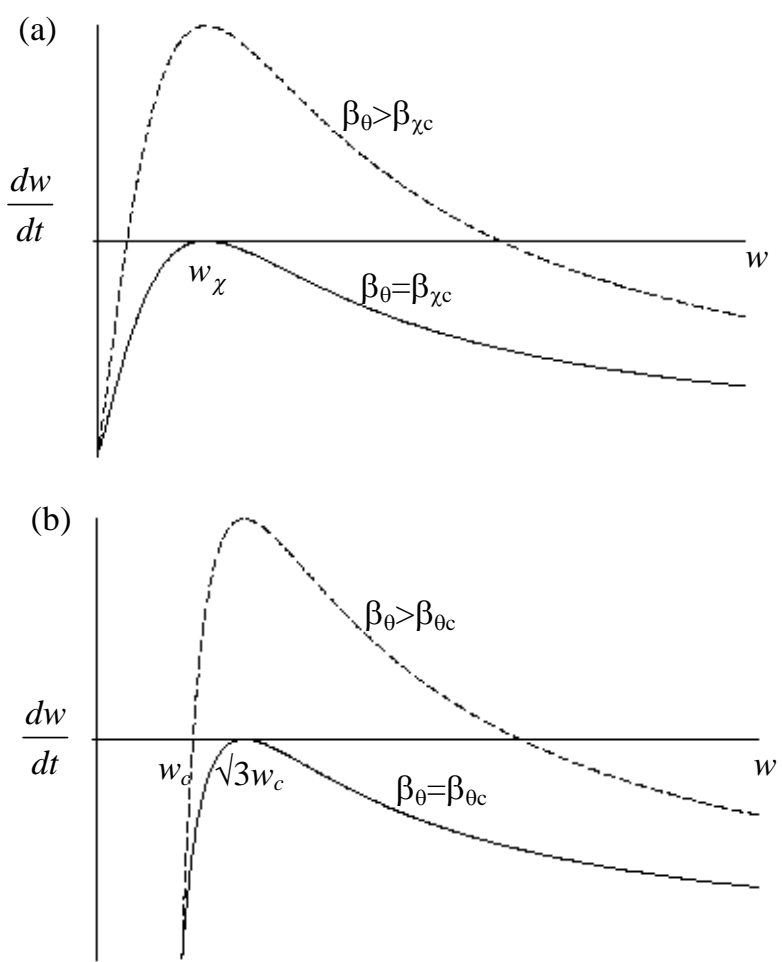

Fig. 3. Plots of $d w / d t$ versus $w$ for (a) the transport threshold model, and (b) the polarisation current model. Curves for $\beta_{\theta}$ equal to its critical value and exceeding this value are shown. 
Note that we have taken account of the effect of the radial transport through a modification of the bootstrap current term: this modification is an interpolation formula, which reproduces $\mathrm{Eq}$ (17) for $w>>w_{\chi}$ and also reproduces the results of linear theory in the opposite limit $w<<w_{\chi}{ }^{5}$. Equation (24) thus provides a model which includes all the essential ingredients of neoclassical tearing modes. [There is an additional, so-called 'Glasser' stabilising term ${ }^{16,17}$, which we have not discussed here due to space limitations; this may be particularly important for spherical toka$\operatorname{maks}^{18}$.]

Both the radial transport effects and the polarisation current can provide a threshold for NTMs. Let us first take $a_{3}=0$ and consider finite $w_{\chi}$ : this is shown in Fig $3 a$ (to be compared with Fig 2, where no threshold effects were included). We see that for $\beta_{\theta}<\beta_{\chi c} d w / d t<0$ for all $w$, so any initial 'seed' perturbation which led to a magnetic island would always decay away. However, for $\beta_{\theta}>\beta_{\chi c}$ the situation is particularly interesting: there are now two values of $w$ for which $d w / d t=0$. For $w<w_{\chi c}, d w / d t<0$ and the island will tend to shrink, while for $w>w_{\chi c}, d w / d t>0$ and the island will grow; indeed it will continue to grow until $w$ reaches $w_{\text {sat }}$, when $d w / d t=0$ again. For $w>w_{\text {sat }}, d w / d t<0$ and islands will decay. Thus we note that $w=w_{\text {sat }}$ again corresponds to a stable point, corresponding to a saturated island. On the other hand, the point $w=w_{\chi c}$ is an unstable point: it corresponds to a threshold in that an initial 'seed' island width must exceed this value for the island to grow to the large width $w=w_{\text {sat }}$. Thus, for this model, two conditions are required for growth of the NTM: both $\beta_{\theta}$ and the 'seed' island width must exceed critical values. These critical values, which can be deduced from Eq (24), are

$$
\beta_{\chi c}=-\frac{2 \Delta^{\prime} w_{\chi}}{a_{2} \sqrt{\varepsilon}} \frac{L_{p}}{L_{q}} \quad w_{\chi c}=\frac{w_{\chi} \beta_{\theta c}}{2 \beta_{\theta}}
$$

where the expression for $w_{\chi c}$ is given for $\beta_{\theta}$ far above threshold (at threshold $w_{\chi c}=w_{\chi}$ ).

We turn to the polarisation term $\left(a_{3} \neq 0\right)$ and set $w_{\chi}=0$. Fig $3 \mathrm{~b}$ shows $d w / d t$ as a function of $w$; we see that it is essentially of the same form as that obtained from the transport effects, described above. Again we see that thresholds in both $\beta_{\theta}$ and $w$ need to be exceeded for island growth, and they can be deduced from Eq (24):

$$
\begin{aligned}
& \beta_{\theta c}=\frac{3 \sqrt{3}}{2 a_{2} \sqrt{\varepsilon}} \frac{L_{p}}{L_{q}}\left(-w_{c} \Delta^{\prime}\right) \\
& w_{c}=\sqrt{\frac{a_{3}}{3 a_{2}}} \frac{\sqrt{g\left(\varepsilon, v_{i}\right)}}{\varepsilon^{1 / 4}} \sqrt{\frac{L_{p}}{L_{q}}} \rho_{b i}
\end{aligned}
$$

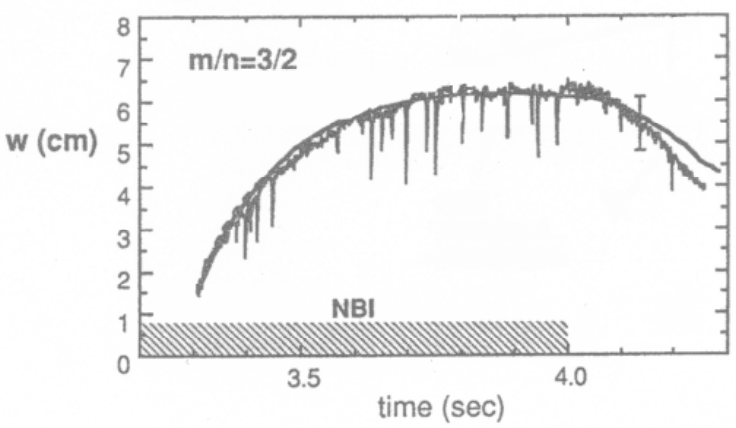

Fig. 4: Trace comparing the experimentally determined island width in TFTR with the result obtained by integrating Eq (17); 'NBI' indicates the time for which neutral beam injection heating was applied [Reprinted with permission from Z Chang et al, Phys Rev Lett 744663 (1995). Copyright (1995) by the American Physical Society.].

The expression for $w_{c}$ is given for $\beta_{\theta}$ far above threshold (at threshold $w_{c}$ is simply a factor $\sqrt{ } 3$ larger). There are two important points to note about this result: (1) the threshold is predicted to be significantly larger in the collisional regime (through the variable $g$, see Eq (25)) and (2) the thresholds are proportional to the ion banana width.

\section{EXPERIMENTAL EVIDENCE}

The first evidence for neoclassical tearing modes in a tokamak came from measurements on the TFTR tokamak $^{19}$. In Fig 4 we show a comparison between the measured magnetic signal and the prediction of Eq (17), and we see that in general the comparison is rather encouraging. However, two features are evident: (1) at the beginning of the trace, we see that the mode is initiated at finite amplitude, suggesting that a threshold $\sim 1 \mathrm{~cm}$ needs to be exceeded for island growth, and (2) the fit is not so good when the island starts to decay. Both of these point towards a

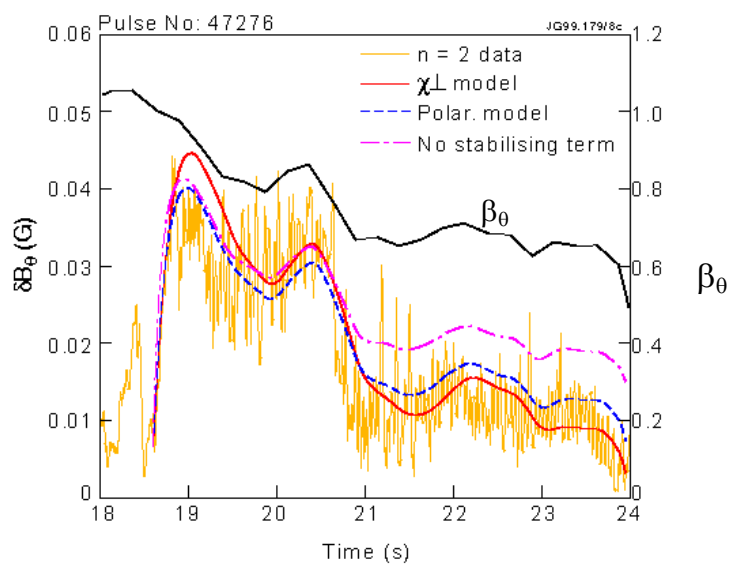

Fig. 5. Tracking the island evolution as the heating power is reduced on JET, we see that inclusion of either of the threshold effects improves the agreement with the measured amplitude of the magnetic perturbation, $\delta B$ (from Ref 20) 
threshold mechanism which is important for small island widths, but has little influence on the evolution of larger islands. Indeed, this is a property of both of the threshold effects we have discussed above. Careful experiments on JET have shown that the agreement between the data and experiment is much better when the threshold effects are taken into account ${ }^{20}$, and this can be seen in Fig 5, where the predicted evolution is plotted (1) neglecting threshold effects, (2) including only the transport effect and (3) including only the polarisation effect. Recent high resolution temperature profile measurements in the vicinity of NTMs on MAST indicate that the transport effects are likely to play a role in the threshold physics ${ }^{21}$.

The theory we have described suggests that very small islands cannot grow (at least if $\Delta^{\prime}<0$ ); ie, island growth cannot occur unless an initial 'seed' island is generated by some other mechanism to excite it above the threshold. This does indeed seem to be the case experimentally, and in many cases NTM growth follows immediately after a sawtooth $\mathrm{crash}^{22,23,24}$. One model is that the sawtooth is predominantly an instability associated with the $q=1$ surface, but that as this instability grows, it induces magnetic island chains at other rational surfaces through toroidal coupling, for example. If these so-called 'sideband' islands exceed the thresholds for NTM growth, then as the sawtooth crash occurs, and the associated $q=1$ instability disappears, the NTM is free to grow. Other types of instability have also been observed to seed NTMs ${ }^{24}$.

Experiments have probed the conditions for NTM onset rather deeply ${ }^{21,25,26}$. In particular, roles have been deduced for both collisionality and $\rho_{*}$ (which is the ratio of ion Larmor radius to minor radius). While there seems no general consensus between the different devices for the dependence on collisionality, it is generally observed that NTMs are only observed at lower values of collisionality. One feature of the polarisation threshold model is that it is a much stronger effect at higher collisionality (through $g\left(\varepsilon, v_{i}\right)$ ), and the transport model can also provide a collisionality dependence. In addition, experiments on ASDEX-Upgrade seemed to confirm a role for $\rho_{*}$ in the threshold $^{25}$, as predicted by the polarisation model, but could also originate from the transport model if one adopts a gyro-Bohm scaling for the perpendicular diffusivity (see $\mathrm{Eq}(23))$. A particular concern for ITER is that a multimachine database appears to indicate that the threshold $\beta_{\theta}$ is linearly proportional to $\rho_{*}$, a parameter which is rather small on ITER ${ }^{20,26}$. On the other hand, there is also some evidence that the seed island size reduces as $\rho_{*}$ gets small$\mathrm{er}^{26}$, and then whether or not NTMs will be an issue on ITER will depend on which gets smaller faster: the threshold, or the seed islands from the sawteeth. So far we do not have sufficiently accurate data in the correct regimes to be confident in the predictions, and therefore it is prudent to assume NTMs will be an issue for ITER, and we must guard against them.

The key to avoiding or controlling NTMs is current drive. One can envisage two schemes: (1) to reduce the free energy available in the equilibrium current profile so that $\Delta^{\prime}$ becomes more negative, and (2) to drive current directly at the island O-point (to replace the missing bootstrap current). Both of these have been tried, with success. In COMPASS-D, radio-frequency waves in the lower hybrid frequency range have been used to drive current close to the rational surface where the island forms ${ }^{23}$. In these experiments, the radial width of the current deposition was typically much wider than the island width, and then it can be shown that there is little contribution to the right hand side of Eq (10). However, calculations of $\Delta^{\prime}$ showed that the additional current that was being driven by the lower hybrid waves did make $\Delta^{\prime}$ more negative, and then both expressions (26) and (27) predict that the threshold for NTMs is increased. Fig 6 shows the experimental results.

The second technique is to drive current directly at the island O-point, highly localised within the magnetic island. Here the stabilisation is achieved through an additional contribution to $J_{\|}$on the right hand side of Eq (10). This has been achieved using radio-frequency waves at the electron-cyclotron resonance, which drives current in a much narrower radial region than the lower hybrid waves used on COMPASS-D. In particular, successful experiments have been performed on ASDEX-Upgrade ${ }^{24}$ and DIII-D ${ }^{25}$, and this is the method envisaged for ITER.

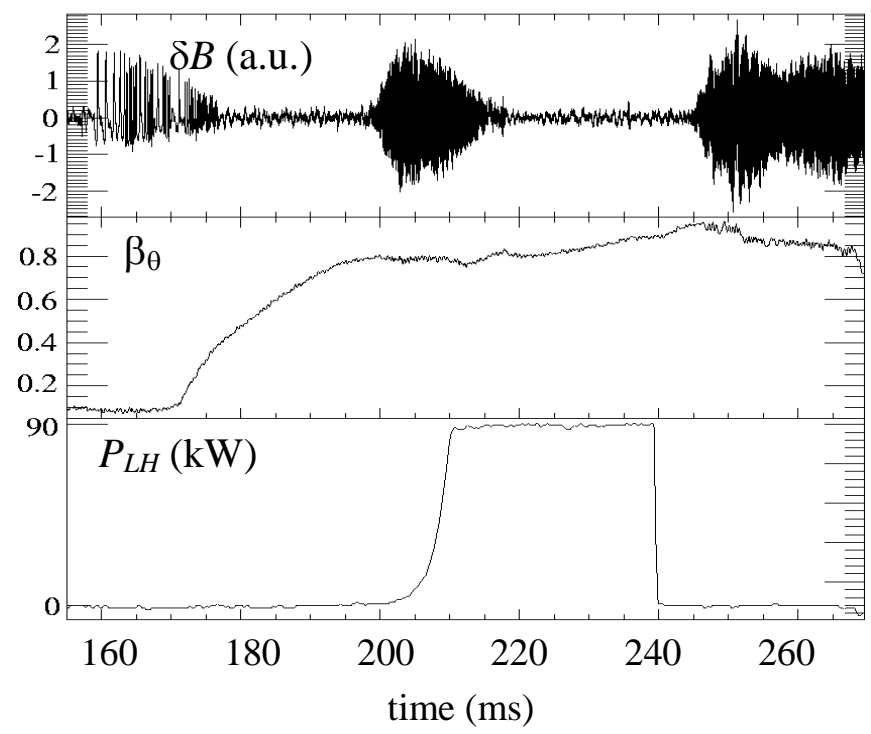

Fig. 6. The magnetic signal $(\delta B)$ shows the growth of a NTM on COMPASS-D after $190 \mathrm{~ms}$, with a corresponding saturation in $\beta_{\theta} .90 \mathrm{~kW}$ of lower hybrid power $\left(\mathrm{P}_{\mathrm{LH}}\right)$ is switched on just after $200 \mathrm{~ms}$, the NTM decays, and $\beta_{\theta}$ again rises. 


\section{SUMMARY}

In summary, understanding the physics of the NTM is one of the success stories of fusion. The instability was predicted 10 years before it was identified experimentally, and since then theories have been refined, and broadly confirmed, by more detailed experiments. Nevertheless, the theory is still some way short of being truly predictive: it needs to address the seed island formation, as well as provide more accurate, quantitative models of the threshold effects, both of which require improved models to describe the relevant situation when the island width is comparable to the ion banana width. This will inevitably require the development of large scale numerical models for the situation. The neoclassical tearing mode is likely to be an issue for ITER, but the prospects for controlling them using radio-frequency waves to drive current close to the rational surface, or perhaps by controlling the seeding mechanism $^{26}$, look promising. This remains an evolving topic of research, both theoretically and experimentally.

\section{ACKNOWLEDGMENTS}

I should like to thank Richard Buttery, Jack Connor and Anthony Webster for their helpful comments.

\section{REFERENCES}

1. $\mathrm{P} \mathrm{H}$ Rutherford "Nonlinear growth of the tearing mode", Phys Fluids 161903 (1973)

2. J Wesson, Tokamaks, first edition (Clarendon Press, 1987), p168

3. R Carrera, R D Hazeltine and M Kotschenreuther "Island bootstrap current modification of the non-linear dynamics of the tearing mode", Phys Fluids 29899 (1986)

4. W X Qu and J D Callen "Nonlinear growth of a single neoclassical MHD tearing mode in a tokamak", University of Wisconsin report UWPR 85-5 (1985)

5. R Fitzpatrick "Helical temperature perturbations associated with tearing modes in tokamak plasmas", Phys Plasmas 2 825 (1995)

6. R D Hazeltine, P Helander and P J Catto "Plasma transport near the separatrix of a magnetic island", Phys Plasmas 42920 (1997)

7. A I Smolyakov "Nonlinear evolution of tearing modes in inhomogeneous plasmas" Plasma Phys Control Fusion 35657 (1993), and references therein.

8. J W Connor, F L Waelbroeck and H R Wilson "The role of polarization current in magnetic island evolution", Phys Plasmas $\underline{8} 2835$ (2001), and references therein

9. H R Wilson, J W Connor, R J Hastie and C C Hegna "Threshold for neoclassical magnetic islands in a low collision frequency tokamak", Phys Plasmas $\underline{3} 248$ (1996)

10. H R Wilson et al "The collisionality dependence of tokamak $\beta$-limits", Plas Phys Control Fusion 38 A149 (1996)
11. F L Waelbroeck, J W Connor and H R Wilson, "Finite Larmor radius theory of magnetic island evolution", Phys Rev Lett 87 (2001) 215003-1

12. A Bergman, E Poli and A G Peeters, "Collisionality dependence of the polarisation current”, Phys Plasmas 12072501 (2005)

13. R. Fitzpatrick and F.L. Waelbroeck, "Drift-tearing magnetic islands in tokamak plasmas" Phys Plasmas 15 (2008) 012502

14. M James, H.R. Wilson and J.W. Connor "Modelling the effect of cross-field diffusion on tearing mode stability”, Plas. Phys. Cont. Fusion 52 (2010) 075008

15. A B Mikhailovskii, et al "An approach to calculation of magnetic island rotation frequency", Phys Plasmas 72530 (2000)

16. M Kotschenreuther, R D Hazeltine and P J Morrison "Nonlinear dynamics of magnetic islands with curvature and pressure", Phys Fluids 28294 (1985)

17. H.Lutjens, et al "Curvature effects on the dynamics of tearing modes in tokamaks, Phys Plasmas 8 (2001) 4267

18. C C Hegna "Nonlinear dynamics of pressure driven magnetic islands in low aspect ratio tokamaks", Phys Plasmas 63980 (1999)

19. Z Chang et al, "Observation of nonlinear neoclassical pressure-gradient-driven tearing modes in TFTR", Phys Rev Lett 744663 (1995)

20. R J Buttery et al "Neoclassical tearing modes", Plasma Phys Control Fusion 42 B61 (2000)

21. J. Snape, et al, "The influence of finite radial diffusion on the structure and evolution of $m / n=2 / 1$ neoclassical tearing modes on MAST", Plas. Phys. Control. Fusion 54 (2011) 085001

22. R J la Haye and O Sauter "Threshold for metastable tearing modes in DIII-D”, Nucl Fusion 38987 (1998)

23. R J Buttery et al "Onset and control of neoclassical tearing modes on JET", Proceedings of the $26^{\text {th }} E P S$ Conference on Controlled Fusion and Plasma Physics (Maastricht) 121 (1999)

24. A Gude et al "Seed island of neoclassical tearing modes at ASDEX Upgrade", Nucl Fusion 39127 (1999)

25. $S$ Günter et al " $\beta$ scaling for the onset of neoclassical tearing modes at ASDEX-Upgrade", Nucl Fusion $\underline{38}$ 1431 (1998)

26. R J la Haye et al "Dimensionless scaling of the critical beta for onset of a neoclassical tearing mode", Phys Plasmas 73349 (2000)

27. C D Warrick et al "Complete stabilization of neoclassical tearing modes with lower hybrid current drive on COMPASS-D", Phys Rev Lett $\underline{85} 574$ (2000)

28. H Zohm et al "Experiments on neoclassical tearing mode stabilization by ECCD in ASDEX Upgrade", Nucl Fusion 39577 (1999)

29. R J la Haye et al "Control of neoclassical tearing modes in DIII-D, Phys Plasmas 92051 (2001)

30. O Sauter et al "Control of neoclassical tearing modes by sawtooth control", Phys Rev Lett $\underline{88}$ 105001-1 (2002) 